\title{
Supplement to The Collatz Conjecture
}

By Anatoliy Nikolaychuk

\section{Abstract}

For the Collatz sequence, we construct the supplement sequence, which is relative to the original sequence. This new method gives a way to find many properties of the sequence, and to prove convergence of the Collatz sequence for all natural numbers.

\section{$1^{\circ}$. Introduction.}

For any natural number $\mathrm{N}$ define the sequence

$a_{n+1}=\left\{\begin{array}{l}\frac{1}{2} a_{n}, \text { if } a_{n} \text { is even } \\ 3 a_{n}+1, \text { if } a_{n} \text { is odd }\end{array}\right.$

Thus, for $\mathrm{N}=5$ we have $a_{0}=5, a_{1}=16, a_{2}=8, a_{3}=4, a_{5}=1, a_{6}=4, \ldots$ and the sequence will repeat itself. The number $\mathrm{N}$ is convergent if corresponding sequence contains 1 .

For the natural number $\mathrm{N}=2^{p} \cdot(2 k+1) \quad(p=0,1,2,3, \ldots ; k=0,1,2,3, \ldots)$ we define the index $\delta(\mathrm{N})$ by the following:

1. $\delta(1)=0$

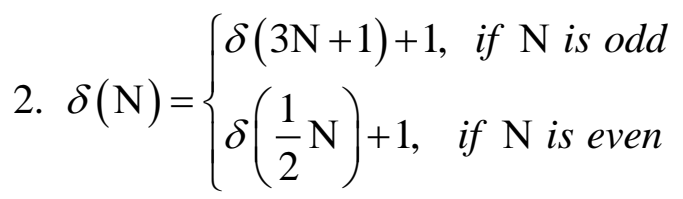

For example, if $\mathrm{N}=7$, the corresponding sequence is $7,22,11,34,17,52,26,13,40,20,10,5,16,8,4,2,1$. Therefore, $\delta(7)=16$, which is the number of steps number 7 goes to 1 .

\section{$2^{\circ}$. The Supplement Sequences.}

For the original sequence corresponding to number $\mathrm{N}$, we may write different supplement sequences, that are convergent together with the original sequence, and all such sequences have the same index.

Example. For the sequence corresponding to $N=26$, we may construct another sequence by the rule:

$$
a_{n+1}= \begin{cases}\frac{a_{n}-1}{2} & \text { if } a_{n} \text { is odd } \\ a_{n} \cdot \frac{3}{2}+1 & \text { if } a_{n} \text { is even }\end{cases}
$$

and we received two "relative" sequences 
$26 \stackrel{\div 2}{\longrightarrow} 13 \stackrel{\times 3+1}{\longrightarrow} 40 \stackrel{\div 2}{\longrightarrow} 20 \stackrel{\div 2}{\longrightarrow} 10 \stackrel{\div 2}{\longrightarrow} 5 \stackrel{\times 3+1}{\longrightarrow} 16 \stackrel{\div 2}{\longrightarrow} 8 \ldots$

$25 \stackrel{-1 \div 2}{\longrightarrow} 12 \stackrel{\times \frac{3}{2}+1}{\longrightarrow} \longrightarrow 19 \stackrel{-1 \div 2}{\longrightarrow} 9 \stackrel{-1 \div 2}{\longrightarrow} 4 \stackrel{\times \frac{3}{2}+1}{\longrightarrow} \longrightarrow 7 \ldots$

or we may construct other sequences

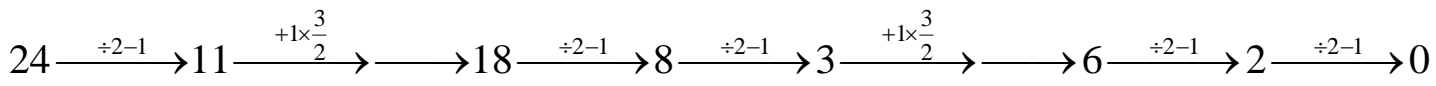

$23 \stackrel{-3 \div 2}{\longrightarrow} 10 \stackrel{\times \frac{3}{2}+2}{\longrightarrow} \longrightarrow 17 \stackrel{-3 \div 2}{\longrightarrow} 7 \stackrel{-3 \div 2}{\longrightarrow} 2 \stackrel{-\times \frac{3}{2}+2}{\longrightarrow} \longrightarrow 5 \stackrel{-3 \div 2}{\longrightarrow} 1 \stackrel{-3 \div 2}{\longrightarrow}-1$

Reader may construct different supplement sequences, by finding appropriate rule,

such that all those sequences are convergent with the same index.

\section{$3^{\circ}$. Basic Supplement Sequence.}

In this article we will consider the supplement sequence defined by the rule:

$a_{n+1}= \begin{cases}\frac{a_{n}+1}{2} & \text { if } a_{n} \text { is odd } \\ a_{n} \cdot \frac{3}{2} & \text { if } a_{n} \text { is even }\end{cases}$

Example: For the number 26 the original and supplement sequences can be written

$$
\begin{aligned}
& 26 \stackrel{\div 2}{\longrightarrow} 13 \stackrel{\times 3+1}{\longrightarrow} 40 \stackrel{\div 2}{\longrightarrow} 20 \stackrel{\div 2}{\longrightarrow} 10 \stackrel{\div 2}{\longrightarrow} 5 \stackrel{\times 3+1}{\longrightarrow} 16 \stackrel{\div 2}{\longrightarrow} 8 \stackrel{\div 2}{\longrightarrow} 4 \stackrel{\div 2}{\longrightarrow} 2 \stackrel{\div 2}{\longrightarrow} 1 \\
& 27 \stackrel{+1 \div 2}{\longrightarrow} 14 \stackrel{\times \frac{3}{2}}{\longrightarrow} \longrightarrow 21 \stackrel{+1 \div 2}{\longrightarrow} 11 \stackrel{+1 \div 2}{\longrightarrow} 6 \stackrel{\times \frac{3}{2}}{\longrightarrow} \longrightarrow 9 \stackrel{+1 \div 2}{\longrightarrow} 5 \stackrel{+1 \div 2}{\longrightarrow} 3 \stackrel{+1 \div 2}{\longrightarrow} 2
\end{aligned}
$$

We see that numbers in the second raw exceed corresponding numbers in the first raw by one. For the new supplement sequence we define the operations:

$A\left(a_{n}\right)=\frac{a_{n}+1}{2}$ if $a_{n}$ is odd and $B\left(a_{n}\right)=a_{n} \cdot \frac{3}{2}$ if $a_{n}$ is even,

and the inverse operations $A^{-1}\left(a_{n}\right)=a_{n} \cdot 2-1, B^{-1}\left(a_{n}\right)=a_{n} \cdot \frac{2}{3}$.

Theorem 3.1 If the natural number $\mathrm{N}$ converges to 1 with index $\delta$ (the number of steps in the original sequence), then corresponding supplement sequence for the number $N+1$ converges to 2 with the same index, where operation $A$ is equivalent to one step, and operation $B$ is equivalent to two steps. Numbers in supplement sequence exceed corresponding numbers in original sequence exactly by 1 .

Proof. a) If $a_{n}$ is odd, then in the original sequence $a_{n} \stackrel{\times 3+1}{\longrightarrow} 3 \cdot a_{n}+1 \stackrel{\div 2}{\longrightarrow} \frac{3 a_{n}+1}{2}$.

For the supplement sequence, $a_{n}+1$ is even, and $a_{n}+1 \stackrel{B}{\longrightarrow} \frac{\left(a_{n}+1\right) \cdot 3}{2}=\frac{3 \cdot a_{n}+1}{2}+1$. 
b) If $a_{n}$ is even, then in the original sequence $a_{n} \stackrel{\div 2}{\longrightarrow} \frac{a_{n}}{2}$, and in the corresponding supplement sequence $a_{n}+1 \stackrel{A}{\longrightarrow} \frac{a_{n}+1+1}{2}=\frac{a_{n}}{2}+1$.

Corollary. The operation A reduces index of a number by 1 , and the operation $B$

reduces index by 2 , whereas inverse operations $A^{-1}$ and $B^{-1}$ increase index by 1 and by 2 correspondingly.

Theorem 3.2. For the even number $\mathrm{N}=4 \mathrm{k}+2(\mathrm{k}=0,1,2,3, \ldots) \quad \delta(4 k+2)=\delta(3 k+2)+3$.

Proof. $4 k+2 \stackrel{B}{\longrightarrow} 6 k+3 \stackrel{A}{\longrightarrow} 3 k+2$, and both operations reduce index totally by 3 .

Corollary. From Theorem 3.2 we see that even number $4 k+2$ is possible to reduce to smaller number $3 k+2$. Any odd number can be reduced in one step using operation $A$.

Therefore, to prove that any natural number converges, it is enough to show this only for numbers which are divisible by 4 .

\section{$4^{\circ}$. Consecutive triples with equal indexes.}

Proposition 4.1. If $\mathrm{k}$ is odd, $k \geq 3$, then $\delta(4 k+2)=\delta(4 k+1)$.

Proof. $4 k+2 \stackrel{B}{\longrightarrow} 6 k+3 \stackrel{A}{\longrightarrow} 3 k+2 \stackrel{A}{\longrightarrow} \frac{3 k+3}{2}$, and

$$
4 k+1 \stackrel{A}{\longrightarrow} 2 k+1 \stackrel{A}{\longrightarrow} k+1 \stackrel{B}{\longrightarrow} \frac{3 k+3}{2} .
$$

We see that the last entries are the same after 4 steps.

Here are some examples of the pair of numbers with equal indexes:

\begin{tabular}{|c|c|c|c|c|c|}
\hline $\mathrm{k}=$ odd & 3 & 5 & $\cdots$ & 21 & $\cdots$ \\
\hline $4 \mathrm{k}+1$ & 13 & 21 & & 85 & \\
\hline $4 \mathrm{k}+2$ & 14 & 22 & & 86 & \\
\hline
\end{tabular}

Proposition 4.2. For any natural number $\mathrm{N}$ and $k=0,1,2,3, \ldots$ $\delta\left(\mathrm{N} \cdot 3^{k} \cdot 32+5\right)=\delta\left(\mathrm{N} \cdot 3^{k} \cdot 32+6\right)=\delta\left(\mathrm{N} \cdot 3^{k} \cdot 32+7\right)$.

Proof. We see the first equality from the following 
$\mathrm{N} \cdot 3^{k} \cdot 32+5 \stackrel{A}{\longrightarrow} \mathrm{N} \cdot 3^{k} \cdot 16+3 \stackrel{A}{\longrightarrow} \mathrm{N} \cdot 3^{k} \cdot 8+2 \stackrel{B}{\longrightarrow} \mathrm{N} \cdot 3^{k} \cdot 12+3$, and

$\mathrm{N} \cdot 3^{k} \cdot 32+6 \stackrel{B}{\longrightarrow} \mathrm{N} \cdot 3^{k} \cdot 48+9 \stackrel{A}{\longrightarrow} \mathrm{N} \cdot 3^{k} \cdot 24+5 \stackrel{A}{\longrightarrow} \mathrm{N} \cdot 3^{k} \cdot 12+3$.

The last entries are the same after 4 steps. We may also recognize that the first

equality is true because $\mathrm{N} \cdot 3^{k} \cdot 32+5=4 \cdot\left(\mathrm{N} \cdot 3^{k} \cdot 8+1\right)+1=4 \cdot($ odd number $)+1$, and $\mathrm{N} \cdot 3^{k} \cdot 32+6=4 \cdot($ odd number $)+2$, and conclusion follows from Proposition 4.1.

The second equality we may see from

$\mathrm{N} \cdot 3^{k} \cdot 32+6 \stackrel{B}{\longrightarrow} \mathrm{N} \cdot 3^{k} \cdot 48+9 \stackrel{A}{\longrightarrow} \mathrm{N} \cdot 3^{k} \cdot 24+5 \stackrel{A}{\longrightarrow} \mathrm{N} \cdot 3^{k} \cdot 12+3 \stackrel{A}{\longrightarrow} \mathrm{N} \cdot 3^{k} \cdot 6+2$

$\stackrel{B}{\longrightarrow} \mathrm{N} \cdot 3^{k} \cdot 9+3$

$\mathrm{N} \cdot 3^{k} \cdot 32+7 \stackrel{A}{\longrightarrow} \mathrm{N} \cdot 3^{k} \cdot 16+4 \stackrel{B}{\longrightarrow} \mathrm{N} \cdot 3^{k} \cdot 24+6 \stackrel{B}{\longrightarrow} \mathrm{N} \cdot 3^{k} \cdot 36+9 \stackrel{A}{\longrightarrow} \mathrm{N} \cdot 3^{k} \cdot 18+5$

$\stackrel{A}{\longrightarrow} \mathrm{N} \cdot 3^{k} \cdot 9+3$

The last entries are the same after 7 steps, therefore, $\delta\left(\mathrm{N} \cdot 3^{k} \cdot 9+3\right)+7=\delta\left(\mathrm{N} \cdot 3^{k} \cdot 32+6\right)=\delta\left(\mathrm{N} \cdot 3^{k} \cdot 32+7\right)$.

Here are some consecutive triples with equal index:

\begin{tabular}{|c|c|c|c|c|}
\hline \multirow{2}{*}{$N$} & 5 & & & \\
& & & & \\
\hline 1 & $7781 ; 7782 ; 7783$ & 23,$333 ; 23,334 ; 23,335$ & 69,$989 ; 69,990 ; 69,991$ & $\ldots$ \\
\hline 12 & 93,$317 ; 93,318 ; 93,319$ & 279,$941 ; 279,942 ; 279,943$ & 839,$813 ; 839,814 ; 839,815$ & $\ldots$ \\
\hline
\end{tabular}

\section{$5^{\circ}$. Sequence of five consecutive numbers with equal indexes.}

Proposition 5.1 For any $k=1,2,3, \ldots \quad \delta(16 k+4)=\delta(16 k+3)$.

Proof.

$$
\begin{aligned}
& 16 k+4 \stackrel{B}{\longrightarrow} 24 k+6 \stackrel{B}{\longrightarrow} 36 k+9 \stackrel{A}{\longrightarrow} 18 k+5 \stackrel{A}{\longrightarrow} 9 k+3 \\
& 16 k+3 \stackrel{A}{\longrightarrow} 8 k+2 \stackrel{B}{\longrightarrow} 12 k+3 \stackrel{A}{\longrightarrow} 6 k+2 \stackrel{B}{\longrightarrow} 9 k+3
\end{aligned}
$$

The last entries are the same in 6 steps, so the statement is proved.

Proposition $5.2 \delta(3 k \cdot 9+2)=\delta(3 k \cdot 9+3)$ if $k$ is odd $(k=1,3,5, \ldots)$ or $k=2+4 n(n=0,1,2, \ldots)$.

Proof. Case 1. If $k=2 n+1=o d d$, then

$3^{2 n+1} \cdot 9+1=3^{p}+1=(3+1)\left(3^{p-1}-3^{p-2}+3^{p-3}-\ldots+1\right)=4 \cdot($ odd number $)$, so 
$3^{k} \cdot 9+2=4 \cdot($ odd number $)+1$, and $3^{k} \cdot 9+3=4 \cdot($ odd number $)+2$. The conclusion follows from the Proposition 4.1.

Case 2. If $k=2+4 n$, then $3^{2+4 n} \cdot 9-1=81^{n+1}-1=(81-1)\left(81^{n}+81^{n-1}+81^{n-2}+\ldots+81+1\right)$

is divisible by 16 . Therefore, $3^{k} \cdot 9+2=16 p+3$ and $3^{k} \cdot 9+3=16 p+4$, and conclusion follows from the Proposition 5.1.

Proposition

$\delta\left(3^{k} \cdot 32+4\right)=\delta\left(3^{k} \cdot 32+5\right)$ if $k=$ odd number $(k=1,3,5, \ldots)$ or $k=2+4 n(n=0,1,2, \ldots)$.

Proof. $3^{k} \cdot 32+4 \stackrel{B}{\longrightarrow} 3^{k+1} \cdot 16+6 \stackrel{B}{\longrightarrow} 3^{k+2} \cdot 8+9 \stackrel{A}{\longrightarrow} 3^{k+2} \cdot 4+5 \stackrel{A}{\longrightarrow} 3^{k+2} \cdot 2+3 \stackrel{A}{\longrightarrow} 3^{k+2}+2$;

$3^{k} \cdot 32+5 \stackrel{A}{\longrightarrow} 3^{k} \cdot 16+3 \stackrel{A}{\longrightarrow} 3^{k} \cdot 8+2 \stackrel{B}{\longrightarrow} 3^{k+1} \cdot 4+3 \stackrel{A}{\longrightarrow} 3^{k+1} \cdot 2+2 \stackrel{B}{\longrightarrow} 3^{k+2}+3$.

The last entries in both cases we get by 7 steps, and by the Proposition 5.2 those entries have the same index .

Theorem 5.1. Five consecutive natural numbers

$3^{k} \cdot 32+3,3^{k} \cdot 32+4,3^{k} \cdot 32+5,3^{k} \cdot 32+6$, and $3^{k} \cdot 32+7$ have the same index if

$k=$ odd number $(k=1,3,5, \ldots)$ or $k=2+4 n(n=0,1,2, \ldots)$.

Proof. This statement for the first two numbers we get by applying Proposition 5.1.

For the last three numbers we have

$$
\begin{aligned}
& 3^{k} \cdot 32+5 \stackrel{A}{\longrightarrow} 3^{k} \cdot 16+3 \stackrel{A}{\longrightarrow} 3^{k} \cdot 8+2 \stackrel{B}{\longrightarrow} 3^{k+1} \cdot 4+3 \stackrel{A}{\longrightarrow} 3^{k+1} \cdot 2+2 \stackrel{B}{\longrightarrow} 3^{k+2}+3 ; \\
& 3^{k} \cdot 32+6 \stackrel{B}{\longrightarrow} 3^{k+1} \cdot 16+9 \stackrel{A}{\longrightarrow} 3^{k+1} \cdot 8+5 \stackrel{A}{\longrightarrow} 3^{k+1} \cdot 4+3 \stackrel{A}{\longrightarrow} 3^{k+1} \cdot 2+2 \stackrel{B}{\longrightarrow} 3^{k+2}+3 ; \\
& 3^{k} \cdot 32+7 \stackrel{A}{\longrightarrow} 3^{k} \cdot 16+4 \stackrel{B}{\longrightarrow} 3^{k+1} \cdot 8+6 \stackrel{B}{\longrightarrow} 3^{k+2} \cdot 4+9 \stackrel{A}{\longrightarrow} 3^{k+2} \cdot 2+5 \stackrel{A}{\longrightarrow} 3^{k+2}+3 .
\end{aligned}
$$

The last entries are the same, and we used 7 steps. By applying Proposition 5.3, we have conclusion of Theorem 5.1.

Example 5.1 Here are some sequences of five consecutive numbers having same index

\begin{tabular}{|c|c|c|}
\hline $\mathrm{k}$ & Consecutive Numbers & Index \\
\hline 1 & $99,100,101,102,103$ & 25 \\
\hline 3 & $867,868,869,870,871$ & 28 \\
\hline 5 & $7779,7780,7781,7782,7783$ & 39 \\
\hline 2 & $291,292,293,294,295$ & 117 \\
\hline
\end{tabular}




\begin{tabular}{|c|c|c|}
\hline 6 & $23331,23332,23333,23334,23335$ & 82 \\
\hline 15 & $459,165,027 ; 459,165,028 ; 459,165,029 ; 459,165,030 ; 459,165,031$ & 134 \\
\hline
\end{tabular}

\section{$6^{\circ}$. Reduction Formulas.}

Theorem 6.1. For any natural number $\mathrm{N}, \delta\left(\mathrm{N} \cdot 2^{p}+m\right)=\delta\left(\mathrm{N} \cdot 3 \cdot 2^{p-2}+m\right)+3$, where $m=2,3,4, \ldots$; and minimum value of $p(p \geq k)$ is given in the following table

Table 6.1

\begin{tabular}{|l|l|l|l|l|l|l|l|l|l|l|l|l|l|l|l|l|}
\hline $\mathrm{m}$ & 2 & 3 & 4 & 5 & 6 & 7 & 8 & 9 & 10 & 11 & 12 & 13 & 14 & 15 & 16 & 17 \\
\hline $\mathrm{k}$ & 2 & 2 & 6 & 3 & 5 & 7 & 12 & 4 & 14 & 6 & 11 & 8 & 8 & 13 & 13 & 5 \\
\hline $\mathrm{m}$ & 18 & 19 & 20 & 21 & 22 & 23 & 24 & 25 & 26 & 27 & 28 & 29 & 30 & 31 & 32 & $\ldots$ \\
\hline $\mathrm{k}$ & 10 & 15 & 15 & 7 & 7 & 12 & 12 & 9 & 17 & 9 & 71 & 14 & 14 & 14 & 68 & $\ldots$ \\
\hline
\end{tabular}

Proof. We will show proof for the value $m=6$. For any other $m$ we do by similar way.

$\mathrm{N} \cdot 2^{p}+6 \stackrel{B}{\longrightarrow} \mathrm{N} \cdot 3 \cdot 2^{p-1}+9 \stackrel{A}{\longrightarrow} \mathrm{N} \cdot 3 \cdot 2^{p-2}+5 \stackrel{A}{\longrightarrow} \mathrm{N} \cdot 3 \cdot 2^{p-3}+3 \stackrel{A}{\longrightarrow} \mathrm{N} \cdot 3 \cdot 2^{p-4}+2$

$\stackrel{B}{\longrightarrow} \mathrm{N} \cdot 3^{2} \cdot 2^{p-5}+3 ;$

$\mathrm{N} \cdot 3 \cdot 2^{p-2}+6 \stackrel{B}{\longrightarrow} \mathrm{N} \cdot 3^{2} \cdot 2^{p-3}+9 \stackrel{A}{\longrightarrow} \mathrm{N} \cdot 3^{2} \cdot 2^{p-4}+5 \stackrel{A}{\longrightarrow} \mathrm{N} \cdot 3^{2} \cdot 2^{p-5}+3$.

In both cases we came to the same number. In the first case we used 7 steps, so

$\delta\left(\mathrm{N} \cdot 2^{p}+6\right)=\delta\left(\mathrm{N} \cdot 3^{2} \cdot 2^{p-5}+3\right)+7$, and in the second case we used 4 steps, so

$\delta\left(\mathrm{N} \cdot 3 \cdot 2^{p-2}+6\right)=\delta\left(\mathrm{N} \cdot 3^{2} \cdot 2^{p-5}+3\right)+4$

Comparing last equalities, we get conclusion of Theorem 6.1 for $\mathrm{m}=6$ and $p \geq 5=\mathrm{k}$.

Now we will try to find longer list of consecutive natural numbers having equal indexes.

In $5^{\circ}$ we found formulas for 5 consecutive numbers with such property and power $p=5$. Table 6.1 shows that we need to increase parameter $p$ to get longer sequence .

Example 6.1. If $\mathrm{p}=6$, we may find easy 6 numbers $3^{10} \cdot 2^{6}+m, \mathrm{~m}=2,3,4,5,6,7$, with equal index $\delta=41$, but $\delta\left(3^{10} \cdot 2^{6}+8\right)=248$. Using Theorem 6.1 and Example 6.1, we have

Theorem 6.2. Six consecutive numbers $3^{10-k} \cdot 2^{6+2 k}+m$, where $\mathrm{m}=2,3,4,5,6,7$ and $\mathrm{k}$ is any fixed number from the set $\{1,2,3,4,5,6,7,8,9,10\}$, have the same index. 
By the other hand, if power of 2 becomes bigger, we may get longer list of consecutive numbers with equal index. For example, if $p=12$, we have 17 consecutive numbers

$3^{7} \cdot 2^{12}+m$, where $\mathrm{m}=2,3, \ldots, 18$ and $\delta=150$. If $\mathrm{p}=14$, we obtain 24 consecutive numbers

$3^{6} \cdot 2^{14}+m$, where $\mathrm{m}=2,3, \ldots, 25$ and $\delta=153$. By increasing parameter $\mathrm{p}$, we receive

Theorem 6.3. 26 consecutive natural numbers $3^{5-k} \cdot 2^{16+2 k}+m$, where $\mathrm{m}=2,3, \ldots, 27$ and $\mathrm{k}$

is any fixed number from the set $\{0,1,2,3,4,5\}$, have the same index $\delta=156+3 k$.

In this article we don't have a goal to find the longest sequence of numbers having the same index. But reader may find such a formulas by increasing parameter $\mathrm{p}$.

\section{$7^{\circ}$. Reduction Theorems.}

We consider now the way to prove that any natural number is convergent. In $3^{\circ}$ we

mentioned that we need to prove this statement for the numbers multiple of four.

We separate all natural numbers by two groups:

group 1: $N=2^{n} \cdot(4 k+1) ;$ group 2: $N=2^{n} \cdot(4 k+3) ; \quad(n, k=0,1,2, \ldots)$.

Lemma 7.1. $\frac{3^{n}+1}{2}\left\{\begin{array}{l}\text { is even, if } \mathrm{n} \text { is odd } \\ \text { is odd, if } \mathrm{n} \text { is even }\end{array}\right.$.

We recognize that because $3^{n}+1$ is divisible by 4 if $n$ is odd, and $3^{n}+1$ is divisible by 2 but not by 4 if $n$ is even.

Theorem 7.1. If $\mathrm{n}$ is even, $n \geq 2$, then for the numbers from group 1 ,

$\delta\left[2^{n}(4 k+1)\right]=\delta\left[2^{n-1}(4 k+1)\right]+1$.

Proof. Using lemma 7.1, we obtain

$2^{n}(4 k+1) \stackrel{B^{n}}{\longrightarrow} 3^{n}(4 k+1) \stackrel{A}{\longrightarrow} 3^{n} \cdot 2 k+\frac{3^{n}+1}{2} \stackrel{A}{\longrightarrow} 3^{n} \cdot k+\frac{3^{n}+3}{4} ;$

$2^{n-1}(4 k+1) \stackrel{B^{n-1}}{\longrightarrow} 3^{n-1}(4 k+1) \stackrel{A}{\longrightarrow} 3^{n-1} \cdot 2 k+\frac{3^{n-1}+1}{2} \stackrel{B}{\longrightarrow} 3^{n} \cdot k+\frac{3^{n}+3}{4}$.

We see that $\delta\left[2^{n}(4 k+1)\right]=\delta\left[3^{n} \cdot k+\frac{3^{n}+3}{4}\right]+(n+2)$, and 
$\delta\left[2^{n-1}(4 k+1)\right]=\delta\left[3^{n} \cdot k+\frac{3^{n}+3}{4}\right]+(n+1)$, which gives the proof of the Theorem 7.1.

Theorem 7.1 shows that $50 \%$ of numbers from group 1 are possible to reduce to twice smaller numbers with index lower by one. Table 7.1 gives list of such "good numbers"

\begin{tabular}{|l|l|l|l|l|l|l|l|l|l|l|l|l|}
\hline \multicolumn{1}{l}{$\mathrm{k}$} & 0 & 1 & 2 & 3 & 4 & 5 & 6 & 7 & 8 & 9 & 10 & $\ldots$ \\
\hline 2 & 4 & 20 & 36 & 52 & 68 & 84 & 100 & 116 & 132 & 148 & 164 & $\ldots$ \\
\hline 4 & 16 & 80 & 144 & 208 & 272 & 336 & 400 & 464 & 528 & 592 & 656 & $\ldots$ \\
\hline 6 & 64 & 320 & 576 & 832 & 1088 & 1344 & 1600 & 1856 & 2112 & 2368 & 2624 & $\ldots$ \\
\hline 8 & 256 & 1280 & 2304 & 3328 & 4352 & 5376 & 6400 & 7424 & 8448 & 9472 & 10,496 & $\ldots$ \\
\hline$\ldots$ & & & & & & & & & & & & \\
\hline
\end{tabular}

Theorem 7.2. If $\mathrm{n}$ is odd $(\mathrm{n}=1,3,5, \ldots)$, then for the numbers from group 2,

$\delta\left[2^{n}(4 k+3)\right]=\delta\left[2^{n-1}(4 k+3)\right]+1$.

Proof. $2^{n}(4 k+3) \stackrel{B^{n}}{\longrightarrow} 3^{n}(4 k+3) \stackrel{A}{\longrightarrow} 3^{n} \cdot 2 k+\frac{3^{n+1}+1}{2} \stackrel{A}{\longrightarrow} 3^{n} \cdot k+\frac{3^{n+1}+3}{4}$;

$2^{n-1}(4 k+3) \stackrel{B^{n-1}}{\longrightarrow} 3^{n-1}(4 k+3) \stackrel{A}{\longrightarrow} 3^{n-1} \cdot 2 k+\frac{3^{n}+1}{2} \stackrel{B}{\longrightarrow} 3^{n} \cdot k+\frac{3^{n+1}+3}{4}$.

The last entries are the same, and $\delta\left[2^{n}(4 k+3)\right]=\delta\left[3^{n} \cdot k+\frac{3^{n+1}+3}{4}\right]+(n+2)$, whereas

$\delta\left[2^{n-1}(4 k+3)\right]=\delta\left[3^{n} \cdot k+\frac{3^{n+1}+3}{4}\right]+(n+1)$, and we get a proof of Theorem 7.2.

Table 7.2 gives some numbers from group 2 that is possible to reduce to twice lower numbers

\begin{tabular}{|l|l|l|l|l|l|l|l|l|l|l|l|l|}
\hline \multicolumn{1}{|c|}{$\mathrm{k}$} & 0 & 1 & 2 & 3 & 4 & 5 & 6 & 7 & 8 & 9 & 10 & $\ldots$ \\
\hline 3 & 24 & 56 & 88 & 120 & 152 & 184 & 216 & 248 & 280 & 312 & 344 & $\ldots$ \\
\hline
\end{tabular}




\begin{tabular}{|l|l|l|l|l|l|l|l|l|l|l|l|l|}
\hline 5 & 96 & 224 & 352 & 480 & 608 & 736 & 864 & 992 & 1120 & 1248 & 1376 & $\ldots$ \\
\hline 7 & 348 & 896 & 1408 & 1920 & 2432 & 2944 & 3456 & 3968 & 4480 & 4992 & 5504 & $\ldots$ \\
\hline$\ldots$ & & & & & & & & & & & & \\
\hline
\end{tabular}

Now we distribute all natural numbers that are multiple of 4 in columns with 3 numbers

in each column and try to drop (take in frame) those which are possible to reduce to lower numbers using Theorems 7.1 and 7.2 .

$\begin{array}{lllllllllllllll}4 & 16 & 28 & 40 & 52 & 64 & 76 & 88 & 100 & 112 & \ldots\end{array}$

$\begin{array}{lllllllllllll}8 & 20 & 32 & 44 & 56 & 68 & 80 & 92 & 104 & 116 & \ldots\end{array}$

$\begin{array}{lllllllllllll}12 & 24 & 36 & 48 & 60 & 72 & 84 & 96 & 108 & 120 & \ldots\end{array}$

Proposition 7.1. For any $\mathrm{k}=0,1,2,3, \ldots \quad \delta(32 k+12)=\delta(27 k+11)+8$, and $\delta(36 k+32)=\delta(32 k+28)-5$.

Proof. $32 k+12 \stackrel{B^{2}}{\longrightarrow} 72 k+27 \stackrel{A}{\longrightarrow} 36 k+14 \stackrel{B}{\longrightarrow} 54 k+21 \stackrel{A}{\longrightarrow} 27 k+11$;

$$
36 k+32 \stackrel{A^{-1}}{\longrightarrow} 72 k+63 \stackrel{B^{-1}}{\longrightarrow} 48 k+42 \stackrel{B^{-1}}{\longrightarrow} 32 k+28 .
$$

Using properties of indexes for the elementary operations, we get conclusions of Proposition 7.1.

Now we may drop more numbers (add frames) in above table, which are possible to reduce to lower numbers using Proposition 7.1. Moreover, we may drop all numbers

in the bottom line 3, because they are divisible by three, and operation $B^{-1}$ reduce such numbers to the lower numbers.

By finding more other reduction formulas similarly like we did in Proposition 7.1, we could try to show that any number in above table can be reduce to lower number. In this article we will demonstrate another method to prove that any natural number is convergent.

\section{$8^{\circ}$. Fundamental Theorem.}

For each natural number $\mathrm{N}$ we will construct the sequence of increasing numbers (not consecutive) having same index as the original number $\mathrm{N}$.

Proposition 8.1. For the numbers from group $1, N=2^{n}(4 k+1)$ when $\mathrm{n}=\mathrm{even}(\mathrm{n}=2,4,6, \ldots)$, 
and for the numbers from group $2, \quad N=2^{n}(4 k+3)$ when $\mathrm{n}=$ odd $(\mathrm{n}=1,3,5, \ldots)$, we have $\delta(N)=\delta(6 N-3)$

Proof. $6 N-3=6 \cdot 2^{n} \cdot($ odd number $)-3 \stackrel{A}{\longrightarrow} 3 \cdot 2^{n} \cdot($ odd number $)-1 \stackrel{A}{\longrightarrow} 3 \cdot 2^{n-1} \cdot($ odd number $)$

$\stackrel{B^{-1}}{\longrightarrow} 2^{n} \cdot($ odd number $)=N$, so $\delta(6 N-3)=\delta(N)+1+1-2$.

Proposition 8.2. For the odd number $N=4 k+3, \mathrm{k}=0,1,2, \ldots, \delta(N)=\delta(6 N-1)$.

Proof. $6 N-1=6(4 k+3)-1=24 k+17 \stackrel{A}{\longrightarrow} 12 k+9 \stackrel{A}{\longrightarrow} 6 k+5 \stackrel{A}{\longrightarrow} 3 k+3 \stackrel{B^{-1}}{\longrightarrow} 2 k+2$ ，

so $\delta(6 N-1)=\delta(2 k+2)+1+1+1-2 ;$ and $N=4 k+3 \stackrel{A}{\longrightarrow} 2 k+2, \delta(N)=\delta(2 k+2)+1$.

Comparing right sides, we get desired conclusion.

Proposition 8.3. $\delta\left[2^{p} \cdot(\right.$ odd number $\left.)+1\right]=\delta\left[2^{p} \cdot(\right.$ odd number $\left.)+1+2^{p-2}\right], \mathrm{p} \geq 2$ ，

$($ odd number $)=3,5,7, \ldots$.

Proof. The number in the left side $2^{p} \cdot($ odd number $)+1 \stackrel{A^{p-2}}{\longrightarrow} 2^{2} \cdot($ odd number $)+1$;

the number in the right side $2^{p} \cdot($ odd number $)+1+2^{p-2} \stackrel{A^{p-2}}{\longrightarrow} 2^{2} \cdot($ odd number $)+1+2^{0}$,

and by the Proposition 4.1, $\delta[4 \cdot($ odd number $)+1]=\delta[4 \cdot($ odd number $)+2]$.

Corollary. If $\mathrm{p}$ is an even number, then $\delta\left[2^{p} \cdot(\right.$ odd number $\left.)+1\right]=\delta\left[2^{p} \cdot(\right.$ odd number $\left.)+1+2^{p-2}+2^{p-4}+\ldots+2^{2}+1\right]$; and

if $p$ is an odd number, then

$\delta\left[2^{p} \cdot(\right.$ odd number $\left.)+1\right]=\delta\left[2^{p} \cdot(\right.$ odd number $\left.)+1+2^{p-2}+2^{p-4}+\ldots+2^{3}+2\right]$.

Proof. The right side in Proposition 8.3

$\delta\left[2^{p}(\right.$ odd number $\left.)+1+2^{p-2}\right]=\delta\left\{2^{p-2}[4(\right.$ odd number $\left.)+1]+1\right\}$, and by applying

Proposition 8.3 again, we get $\delta\left[2^{p}(\right.$ odd number $\left.)+1+2^{p-2}+2^{p-4}\right]$, and continue by induction, we get desire conclusion.

Definition. Define the following operations:

$P(N)=6 N-3, Q(N)=6 N-1, \quad R\left[2^{p}(\right.$ odd number $\left.)+1\right]=2^{p}($ odd number $)+1+2^{p-2}$. 
Now we will use those operations to construct for the natural number $\mathrm{N}$ corresponding sequence of increasing numbers having the same index.

Example 8.1. For $\mathrm{N}=20$, using operations $\mathrm{A}, \mathrm{B}$, we get the sequence

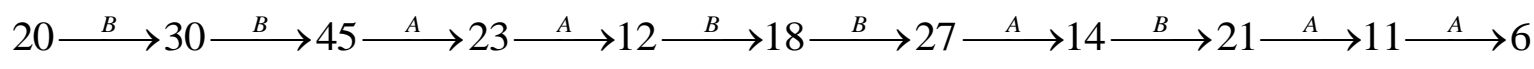

$\stackrel{B}{\longrightarrow} 9 \stackrel{A}{\longrightarrow} 5 \stackrel{A}{\longrightarrow} 3 \stackrel{A}{\longrightarrow} 2$.

But using operations $P, Q$, and $R$, we get the sequence

$$
\begin{aligned}
& 20 \stackrel{P}{\longrightarrow} 117\left(2^{2} \cdot 29+1\right) \stackrel{R}{\longrightarrow} 118 \stackrel{P}{\longrightarrow} 705\left(2^{6} \cdot 11+1\right) \stackrel{R^{3}}{\longrightarrow} 726 \stackrel{P}{\longrightarrow} 4353\left(2^{8} \cdot 17+1\right) \\
& \stackrel{R^{4}}{\longrightarrow} 4438 \stackrel{P}{\longrightarrow} 26,625\left(2^{11} \cdot 13+1\right) \stackrel{R^{5}}{\longrightarrow} 27,307 \stackrel{Q}{\longrightarrow} 163,841\left(2^{15} \cdot 5+1\right) \stackrel{R^{7}}{\longrightarrow} 174,763 \\
& \stackrel{Q}{\longrightarrow} 1,048,577\left(2^{20}+1\right) .
\end{aligned}
$$

Let us analyze increasing numbers in this sequence. According to Propositions 8.1, 8.2, and 8.3, all those numbers have the same index. The last number $2^{20}+1$ has index $\delta=20$, because $2^{20}+1 \stackrel{A^{20}}{\longrightarrow} 2^{0}+1=2$ . Obviously, the number $2^{20}+1$ is the greatest natural number having index 20 . Further increasing this number changes index,

$2^{20} \cdot 1+1 \stackrel{R}{\longrightarrow} 2^{20} \cdot 1+1+2^{18}=2^{18} \cdot 5+1 \stackrel{A^{18}}{\longrightarrow} 2^{0} \cdot 5+1=6$, therefore, $\delta\left(2^{20}+1+2^{18}\right)=18+\delta(6)=18+5=23$. That's why in Proposition 8.3, (odd number) should be greater than 1.

Now we consider increasing numbers in our sequence:

$117=2^{2} \cdot 29+1 \stackrel{A^{2}}{\longrightarrow} 2^{0} \cdot 29+1=30$

$705=2^{6} \cdot 11+1 \stackrel{A^{6}}{\longrightarrow} 2^{0} \cdot 11+1=12 ;$

$4353=2^{8} \cdot 17+1 \stackrel{A^{8}}{\longrightarrow} 2^{0} \cdot 17+1=18 ;$

$26,625=2^{11} \cdot 13+1 \stackrel{A^{11}}{\longrightarrow} 2^{0} \cdot 13+1=14$;

$163,841=2^{15} \cdot 5+1 \stackrel{A^{15}}{\longrightarrow} 2^{0} \cdot 5+1=6 ;$

$1,048,577=2^{20}+1 \stackrel{A^{20}}{\longrightarrow} 2^{0}+1=2$.

We may see that numbers $30,12,18,14,6,2$ are the same even numbers in regular convergent sequence corresponding $\mathrm{N}=20$. Therefore, we have relation between two convergent sequences. New constructed sequence satisfy the following properties:

a) sequence is increasing and bounded,

b) all numbers in the sequence have the same index, 
c) the last (biggest) number in the sequence equals $2^{n}+1$, where $n=\delta(N)$.

Example 8.2. For $\mathrm{N}=40$ we have the sequence

$$
\begin{aligned}
& 40 \stackrel{Q}{\longrightarrow} 239 \stackrel{Q}{\longrightarrow} 1433\left(2^{3} \cdot 179+1\right) \stackrel{R}{\longrightarrow} 1435 \stackrel{Q}{\longrightarrow} 8609\left(2^{5} \cdot 269+1\right) \stackrel{R^{2}}{\longrightarrow} 8619 \\
& \stackrel{Q}{\longrightarrow} 51,713\left(2^{9} \cdot 101+1\right) \stackrel{R^{4}}{\longrightarrow} 51,883 \stackrel{Q}{\longrightarrow} 311,297\left(2^{14} \cdot 19+1\right) \stackrel{R^{7}}{\longrightarrow} 316,758 \\
& \stackrel{P}{\longrightarrow} 1,900,545\left(2^{16} \cdot 29+1\right) \stackrel{R^{8}}{\longrightarrow} 1,922,390 \stackrel{P}{\longrightarrow} 11,534,337\left(2^{20} \cdot 11+1\right) \stackrel{R^{10}}{\longrightarrow} 11,883,862 \\
& \stackrel{P}{\longrightarrow} 71,303,169\left(2^{22} \cdot 17+1\right) \stackrel{R^{11}}{\longrightarrow} 72,701,270 \stackrel{P}{\longrightarrow} 436,207,617\left(2^{25} \cdot 13+1\right) \\
& \stackrel{R^{12}}{\longrightarrow} 447,392,427 \stackrel{Q}{\longrightarrow} 2,684,354,561\left(2^{29} \cdot 5+1\right) \stackrel{R^{14}}{\longrightarrow} 2,863,311,531 \stackrel{Q}{\longrightarrow} 17,179,869,185 \\
& =2^{34}+1 . \text { Therefore, } \delta(40)=34 .
\end{aligned}
$$

Collecting information in $8^{\circ}$, we may write

Theorem 8.1 (Fundamental). For any natural number $\mathrm{N}>2$, we may construct the sequence using operations $P, Q, R$, which satisfy the following conditions:
a) sequence is increasing and bounded,
b) all numbers in the sequence have the same index,
c) the biggest number in the sequence is $2^{n}+1$, where $n=\delta(N)$.

\section{References:}

1. O'Connor, J.J.; Robertson, E.F. (2006). "Lothar Collatz". St Andrews University School of Mathematics and Statistics, Scotland.

2. Maddux, Cleborne D.; Johnson, D. Lamont (1997). Logo: A Retrospective. New York: Haworth Press. p. 160. ISBN 0-7890-0374-0. The problem is also known by several other names, including: Ulam's conjecture, the Hailstone problem, the Syracuse problem, Kakutani's problem, Hasse's algorithm, and the Collatz problem.

3. Lagarias, Jeffrey C. (1985). "The $3 x+1$ problem and its generalizations". The American Mathematical Monthly. 92 (1): 3-23. JSTOR 2322189.

4. Pickover, Clifford A. (2001). Wonders of Numbers. Oxford: Oxford University Press. pp. 116-118. ISBN $\underline{\text { O- }}$ 19-513342-0.

5. Guy, Richard K. (2004). "'E17: Permutation Sequences"'". Unsolved problems in number theory (3rd ed.). Springer-Verlag. pp. 336-7. ISBN $\underline{Z b l} \underline{1058.11001 .}$

6. Guy, R. K. (1983). "Don't try to solve these problems". Amer. Math. Monthly. 90: 3541. doi:10.2307/2975688. JSTOR 2975688. By this Erdos means that there aren't powerful tools for manipulating such objects. 
7. Lagarias, Jeffrey C., ed. (2010). The ultimate challenge: the $3 x+1$ problem. Providence, R.I.: American Mathematical Society. p. 4. ISBN $\underline{0821849409 .}$

8. Leavens, Gary T.; Vermeulen, Mike (December 1992). "3x+1 Search Programs". Computers \& Mathematics with Applications. 24 (11): 79-99. doi:10.1016/0898-1221(92)90034-F.

9. Roosendaal, Eric. "3x+1 Delay Records". Retrieved 30 June 2017. (Note: "Delay records" are total stopping time records.)

10. Steiner, R. P. (1977). "A theorem on the syracuse problem". Proceedings of the 7th Manitoba Conference on Numerical Mathematics. pp. 553-9. MR $\underline{0535032 .}$

11. Colussi, Livio (9 September 2011). "The convergence classes of Collatz function". Theoretical Computer Science. 412 (39): 5409-5419. doi:10.1016/j.tcs.2011.05.056.

12. Terras, Riho (1976), "A stopping time problem on the positive integers" (PDF), Polska Akademia Nauk, 30 (3): 241-252, doi: 10.4064/aa-30-3-241-252, MR 0568274.

13. Kurtz, Stuart A.; Simon, Janos (2007). "The Undecidability of the Generalized Collatz Problem". In Cai, J.Y.; Cooper, S. B.; Zhu, H. Proceedings of the 4th International Conference on Theory and Applications of Models of Computation, TAMC 2007, held in Shanghai, China in May 2007. pp. 542553. doi: $10.1007 / 978-3-540-72504-6$ 49. ISBN 3-540-72503-2. As PDF. 